\title{
Sub-Ångstrom Resolution with Aberration-Corrected TEM: Present and Future
}

\author{
Michael A. O’Keefe
}

Materials Sciences Division, LBNL 2-200, 1 Cyclotron Road, Berkeley, CA 94720, USA

DISCLAIMER

This document was prepared as an account of work sponsored by the United States Government. While this document is believed to contain correct information, neither the United States Government nor any agency thereof, nor The Regents of the University of California, nor any of their employees, makes any warranty, express or implied, or assumes any legal responsibility for the accuracy, completeness, or usefulness of any information, apparatus, product, or process disclosed, or represents that its use would not infringe privately owned rights. Reference herein to any specific commercial product, process, or service by its trade name, trademark, manufacturer, or otherwise, does not necessarily constitute or imply its endorsement, recommendation, or favoring by the United States Government or any agency thereof, or The Regents of the University of California. The views and opinions of authors expressed herein do not necessarily state or reflect those of the United States Government or any agency thereof, or The Regents of the University of California.

Ernest Orlando Lawrence Berkeley National Laboratory is an equal opportunity employer.

Ernest Orlando Lawrence Berkeley National Laboratory - LBNL-55694 


\section{Sub-Ångstrom Resolution with Aberration-Corrected TEM: Present and Future}

Michael A. O'Keefe

Materials Sciences Division, LBNL 2-200, 1 Cyclotron Road, Berkeley, CA 94720, USA

Resolution is the ability to determine if a feature in an image represents two objects rather than one. Rayleigh's resolution criterion [1], an accepted standard in optics, was derived as a means for judging when two sources of light (stars) were distinguishable from a single source. In microscopy, resolution is the ability to determine if detail in an image represents distinct (separated) objects. In high-resolution TEM, these objects are atoms. Resolution of $|\mathbf{d}|$ is achieved when atoms separated by a (projected) distance $|\mathbf{d}|$ can be perceived as separate objects. Presence of the $1 /|\mathbf{d}|$ frequency in the TEM image spectrum is not sufficient to demonstrate a corresponding resolution of $|\mathbf{d}|[2]$. The standard test of TEM image resolution should be the separation of atom peaks in the image [3].

In the high-resolution TEM, the sample is oriented along a relatively low-index zone axis where the atom columns to be imaged are well separated in projection. The incident electron beam interacts strongly with the crystal, forming multiple diffracted beams. These diffracted beams are brought together by the objective lens so that they can interfere to create an image. TEM images are able to depict the projected atom columns because they are interference patterns of the directly transmitted beam with beams diffracted from the specimen imaged under well-established conditions.

Structural information from the specimen is encoded in the phase of the scattered electron waves [4]. Although the electron phase is not an observable (it is not gauge invariant [5]), phase differences can be measured by interference experiments. A direct way is by electron holography [6], but the usual method is to image at the "optimum" or "extended Scherzer" defocus [7]. Then objective lens phase shifts allow interference of the scattered electron waves exiting the specimen to turn the relative phases of the waves into image peaks mapping the atom positions (at the resolution of the microscope). This result has been verified by theory [8], simulation [9], and countless experiments. The native, or Scherzer, resolution limit of the uncorrected high-resolution transmission electron microscope is $d_{X}=0.64 C_{S}{ }^{1 / 4} \lambda^{3 / 4}$ where $C_{S}$ is the spherical aberration coefficient and $\lambda$ the electron wavelength $[7,10]$. Increasing the accelerating voltage (and the cost) improves resolution by reducing electron wavelength. Objective pole-piece saturation keeps the product of $\mathrm{C}_{S}$ and $\lambda$ (almost) constant with increasing voltage, so that a wavelength of $\lambda(\AA)$ will allow a resolution $d(\AA)$ of 12 times $\sqrt{\lambda}$. Mid-voltage TEMs can achieve $1.9 \AA$ resolution at $200 \mathrm{kV}(1.7 \AA$ at $300 \mathrm{kV})$, but require aberration correction $\left(\mathrm{C}_{\mathrm{S}}\right.$ reduction) to reach sub-Angstrom levels [11]. $\mathrm{C}_{\mathrm{S}}$ can be reduced directly (hardware correction [12]), or by using several images (with phase changes known from objective lens defocus) to compute the phase of the electron wave exiting the specimen (software correction [13]).

Hardware and software correction have each produced sub-Ångstrom images [10,14], and imaged light atoms such as oxygen $[15,16]$. The One-Ångstrom Microscope $(\mathrm{OAM})$ combines a modified CM300FEG/UT TEM with FEI focal-series reconstruction (TrueImage ${ }^{\mathrm{TM}}$ ) software by Coene and Thust $[17,18]$ to achieve sub- $\AA$ resolution to $0.78 \AA$ [19]. Modifications include hardware correction of 3-fold astigmatism to $0.68 \AA$ and information limit extension to $0.78 \AA$ [10]. The OAM can image atoms as light (small) as nitrogen [20], carbon [10], and lithium [21]. OAM results presage those to come from hardware-corrected TEMs. Advances in technology, including new electron-beam monochromators, higher-stability high-voltage power supplies, and objective lens power supplies, should be able to improve the TEM information limit by a factor of two or so over the OAM's.

Focal-series reconstruction (FSR) is more than just $\mathrm{C}_{S}$ correction. Its compensation of imperfect objective lens transfer provides improvement over any single image. Single images of diamond and silicon (Fig. 1) show carbon atoms clearly separated by $0.89 \AA$ and (at the information limit of the OAM) silicon atoms separated by $0.78 \AA$. C $\mathrm{C}_{\mathrm{S}}$-corrected OAM images (Fig. 2), assembled from 20member focal series, are "cleaner", due to lack of second-order contributions. Note that secondorder components can be removed from single images by subtracting a minimum-contrast image [22] (at zero defocus for the case of zero $\mathrm{C}_{\mathrm{S}}$ ), thus extending the interpretable specimen thickness [23] as for focal-series reconstruction. Comparison of figures 1 and 2 shows that TEM images are able to depict atom positions just as well as do FSR images, provided both have the same resolution [24]. 


\section{References}

[1] Lord Rayleigh, Philosophical Magazine 47, 310: (1874) 81-93.

[2] M.A. O'Keefe, 37th Ann. Proc. EMSA, San Antonio, Texas (1979) 556-557.

[3] M.A. O'Keefe \& L.F. Allard, Microscopy \& Microanalysis 10, 2: (2004) these proceedings.

[4] J.M. Cowley, Diffraction Physics (1975) North Holland / American Elsevier.

[5] H. Rose, Lectures on Charged Particle Optics, LBNL (2004)

[6] H. Lichte, Ultramicroscopy 38 (1991) 13-22.

[7] O. Scherzer, J. Appl. Phys. 20 (1949) 20-29.

[8] J.M. Cowley \& S. Iijima, Z. Naturforsch. 27a (1972) 445-451.

[9] M.A. O'Keefe, P.R. Buseck \& S. Iijima, Nature 274 (1978) 322-324.

[10] M.A. O'Keefe et al., Ultramicroscopy 89, 4: (2001) 215-241.

[11] H. Rose, Ultramicroscopy 56 (1994) 11-25.

[12] M. Haider \& S. Uhlemann, Proc. 55th Ann. Meeting EMSA, Cleveland, Ohio (1997) 1179-1180.

[13] P. Schiske, Image Processing And Computer-Aided Design (1973) Ed. P.W. Hawkes, pp.82-90.

[14] J.L. Hutchison et al., JEOL News 37E (2002) 2-5.

[15] C.L Jia, M. Lentzen, \& K. Urban, Science 299 (2003) 870-873.

[16] C.L. Jia, \& A. Thust, Phys. Rev. Letts 82 (1999) 5052-5055.

[17] W.M.J. Coene, A. Thust, M. Op de Beeck \& D. Van Dyck, Ultramicroscopy 64 (1996) 109-135.

[18] A. Thust, W.M.J. Coene, M. Op de Beeck \& D. Van Dyck, Ultramicroscopy 64 (1996) 211-230.

[19] M.A. O'Keefe, E.C. Nelson, Y.C. Wang \& A. Thust, Philosophical Mag. B 81 (2001) 11, 1861-1878.

[20] C. Kisielowski et al., Ultramicroscopy 89 (2001) 4: 243-263.

[21] Y. Shao-Horn, L. Croguennec, C. Delmas, E.C. Nelson \& M.A. O’Keefe, Nature Mat. 2 (2003) 464-467

[22] M.A. O'Keefe \& J.V. Sanders, Optik 46 (1976) 421-430.

[23] M.A. O’Keefe, Microscopy \& Microanalysis 4, supple. 2: (1998) 382-383.

[24] Supported by Director, Office of Science, Office of Basic Energy Sciences, Materials Science Division, US Dept. of Energy under contract DE-AC03-76SF00098. Microscopy carried out at the NCEM, LBNL.
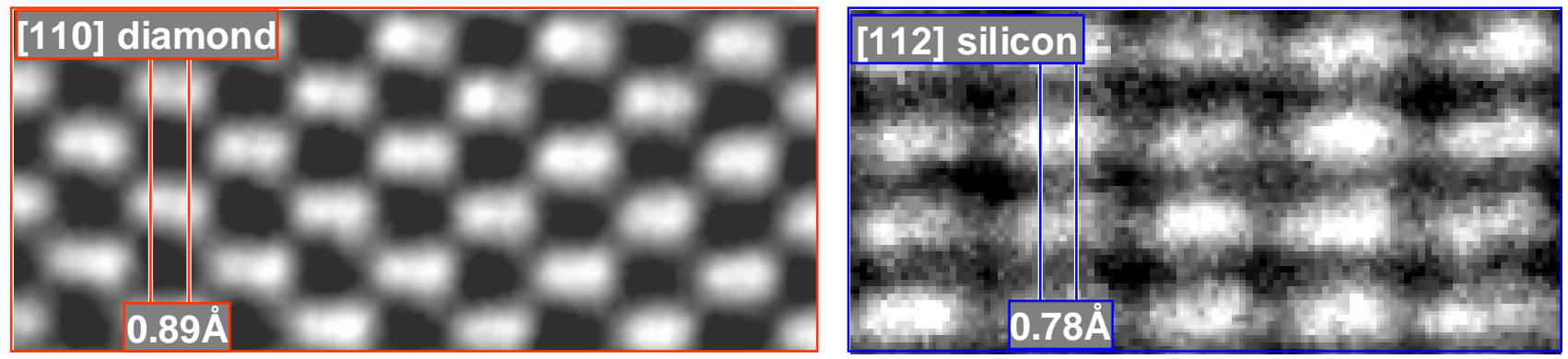

FIG. 1. Single-shot images obtained close to alpha-null defocus [10], shown at magnifications of 50 million times. Left image shows $0.89 \AA$ carbon atom spacing in [110] diamond [10]. Right image shows $0.78 \AA$ silicon atom spacing in [112] silicon at the OAM resolution limit [19].
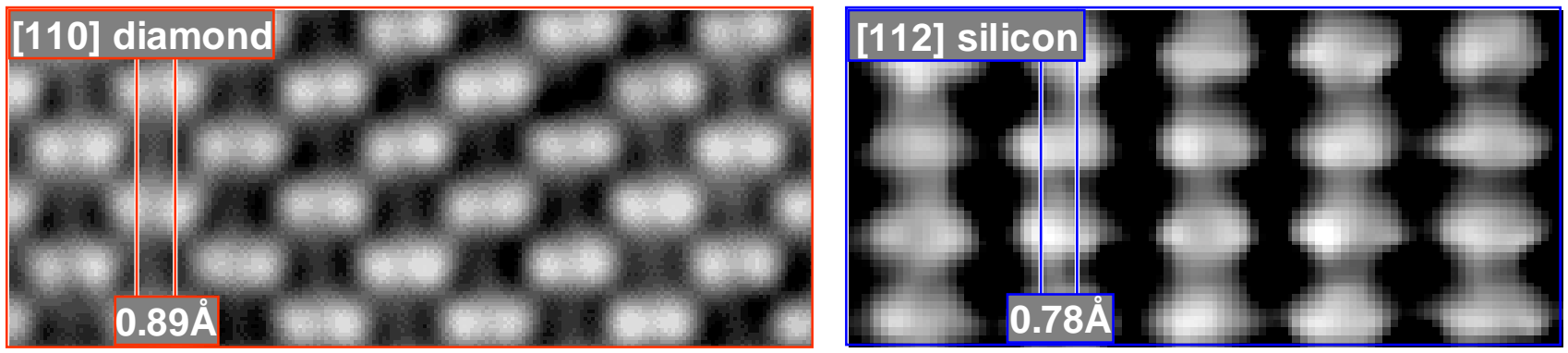

FIG. 2. OÅM images, aberration-corrected by reconstruction from focal-series of 20 images, shown at 50 million times magnification. Left image shows $0.89 \AA$ carbon atom spacing in [110] diamond [10]. Right image shows $0.78 \AA$ silicon atom spacing in [112] silicon. Focal-series-reconstructed images appear "cleaner" than single-shot images due to lack of "second-order interferences" [2]. 